\title{
An abrupt-onset shoulder joint subluxation and pseudoparalysis caused by intraarticular pigmented villonodular synovitis: A case report
}

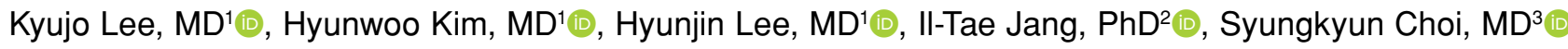 \\ ${ }^{1}$ Nanoori 2bko R\&D Institute, Nanoori Hospital Suwon, Suwon, Republic of Korea \\ ${ }^{2}$ Department of Neurosurgery, Nanoori Hospital Gangnam, Seoul, Republic of Korea \\ ${ }^{3}$ Department of Orthopedic Surgery, Nanoori Hospital Gangnam, Seoul, Republic of Korea
}

Pigmented villonodular synovitis (PVNS) is a rare, idiopathic, synovial proliferative disease with a reported incidence rate of $0.0002 \% \cdot{ }^{[1,2]}$ It mostly affects the knee and fingers. It is classified into two main types as localized and diffuse and the localized form is found as a nodular or pedunculated lesion, while the diffuse form involves the entire synovial membrane of the affected joints. ${ }^{[2]}$ The disease usually occurs among those aged 30 to 59 years and males and females are affected at equal rates. It usually involves a single joint, most commonly the knee joint in $80 \%$ of cases. ${ }^{[3]}$ Hemarthrosis and Charcot's neuropathic arthritis can be considered in the differential diagnosis. It rarely occurs in the shoulders, with fewer than 50 cases reported in the literature to date. ${ }^{[1-4]}$

\section{Received: May 21, 2020}

Accepted: September 11, 2020

Published online: January 06, 2021

Correspondence: Syungkyun Choi, MD. Department of Orthopedic Surgery, Nanoori Hospital Gangnam, 63-8

Nonhyun-Dong, Gangnam-gu, Seoul 06048, Republic of Korea.

E-mail: bluetear0616@naver.com

Doi: 10.5606/ehc.2021.75437

Citation: Lee $\mathrm{K}$, Kim H, Lee $\mathrm{H}$, Jang II-T, Choi S. An abruptonset shoulder joint subluxation and pseudoparalysis caused by intraarticular pigmented villonodular synovitis: A case report. Jt Dis Relat Surg 2021;32(1):258-261.

○2021 All right reserved by the Turkish Joint Diseases Foundation

This is an open access article under the terms of the Creative Commons Attribution-NonCommercial License, which permits use, distribution and reproduction in any medium, provided the original work is properly cited and is not used for commercial purposes (http://creativecommons.org/licenses/by-nc/4.0/).

\section{ABSTRACT}

Pigmented villonodular synovitis (PVNS) occurs most frequently in the knee and hand joints and is extremely rare in the shoulder joints. A 27-year-old women was admitted to our outpatient clinic with an abrupt-onset pain and limited range of motion of the left shoulder. On arthroscopic examination, localized PVNS surrounding the reactive synovitis in the posterior aspect of the subscapularis within the shoulder joint cavity. The mass was removed using a grasper and shaver. Symptomatic relief was achieved following surgical removal. In conclusion, although rare, localized PVNS of the shoulder joint should be kept in mind in patients with a sudden-onset shoulder joint subluxation and pseudoparalysis.

Keywords: Arthroscopy, pigmented villonodular synovitis, shoulder.

In this article, we report a rare case of localized PVNS presenting with an abrupt-onset shoulder joint subluxation and pseudoparalysis.

\section{CASE REPORT}

A 27-year-old female patient was presented to our outpatient clinic with complaints of pain and limited range of motion (ROM) which suddenly occurred, starting a day earlier. Physical examination revealed mild swelling and edema in the left shoulder region. The patient appeared to have upper limb paralysis due to pain and limited active and passive ROM of the shoulder joint. Sensation, blood circulation, and motion in the distal part of the left upper arm were all intact. However, severe tenderness and edema were observed in the subscapularis muscle with the left shoulder in the externally rotated position. Blood test results showed that the leukocyte count, platelet count, blood coagulation test, erythrocyte sedimentation rate, C-reactive protein levels, and 
rheumatoid arthritis factors were all normal. In the radiological examination, anteroposterior images showed widening of the glenohumeral joint space and inferior subluxation (Figure 1), based on which joint edema was suspected. On magnetic resonance imaging (MRI), axial images showed increased joint fluid and thickening of the synovial membrane in the shoulder. A well-capsulated mass, approximately $3 \times 3 \mathrm{~cm}$ in size, was observed in the anterior aspect of the subscapularis muscle (Figure 2). A heterogeneous intermediate-to-high intensity signal was seen on the T2 image and an intermediate intensity signal was seen on the T1 image. The pedunculated mass appeared to originate from an area between the anterior inferior labrum and anterior capsule on axial images. Arthroscopic surgery was decided and a written informed consent was obtained from the patient.

Arthroscopic surgery was performed in the lateral position under interscalene block and intravenous sedation. Intraoperative findings revealed hemarthrosis. Once the intraarticular space was accessed via a posterior portal approach, a large, pedunculated, well-capsulated movable mass was observed between the subscapularis muscle and anterior labrum (Figure 3). Consistent with the MRI findings, the origin of the mass was confirmed to be in the area between the anterior inferior labrum

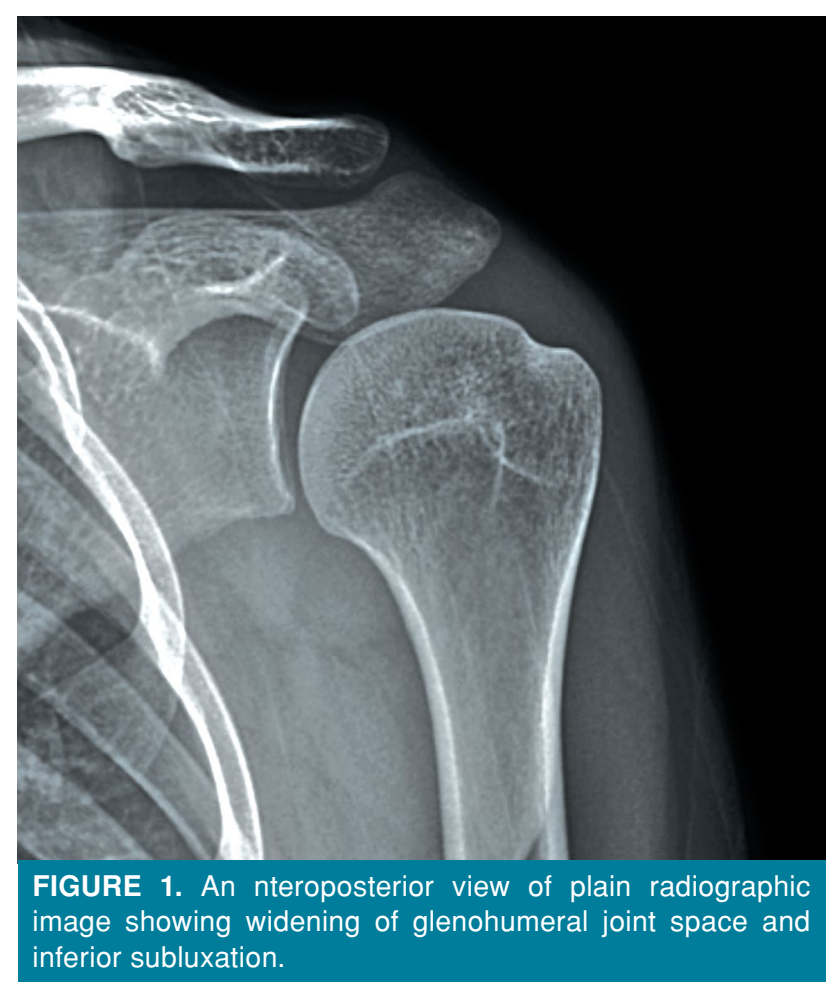

and anterior capsule, while reactive synovitis was observed in the periphery. The mass was removed using a grasper, while the peripheral synovitis and lesions appearing to be residual masses were removed using a shaver. Fraying was found in the cartilage of the humeral head that was impinged by the mass. A partial anterior labral tear was observed, but was not considered to require suturing. On histological examination, hematoxylin and eosin (H-E) staining revealed fibroblasts and giant cells, along with

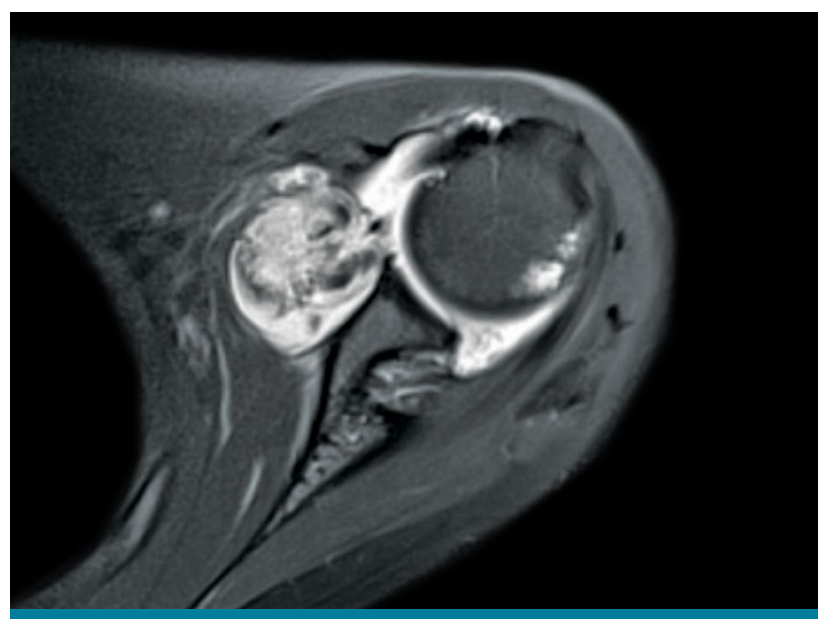

FIGURE 2. T2 axial image of magnetic resonance imaging showing increased joint fluid with thickening of synovial membrane. A $3 \times 3-\mathrm{cm}$ sized, well-capsulated lesion was observed in anterior aspect of subscapularis muscle.

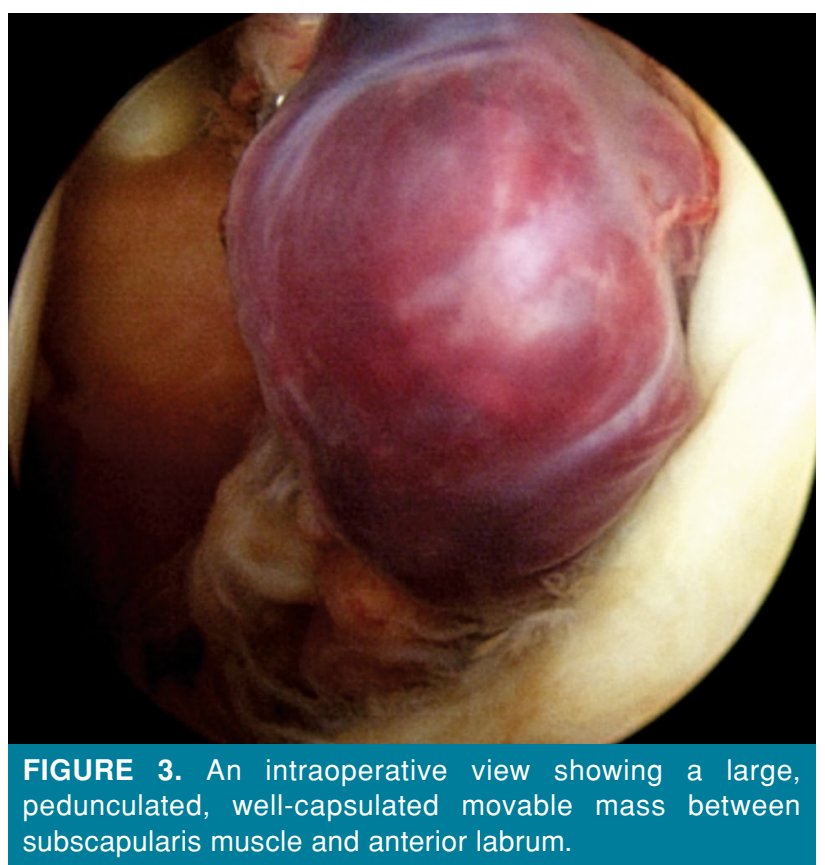




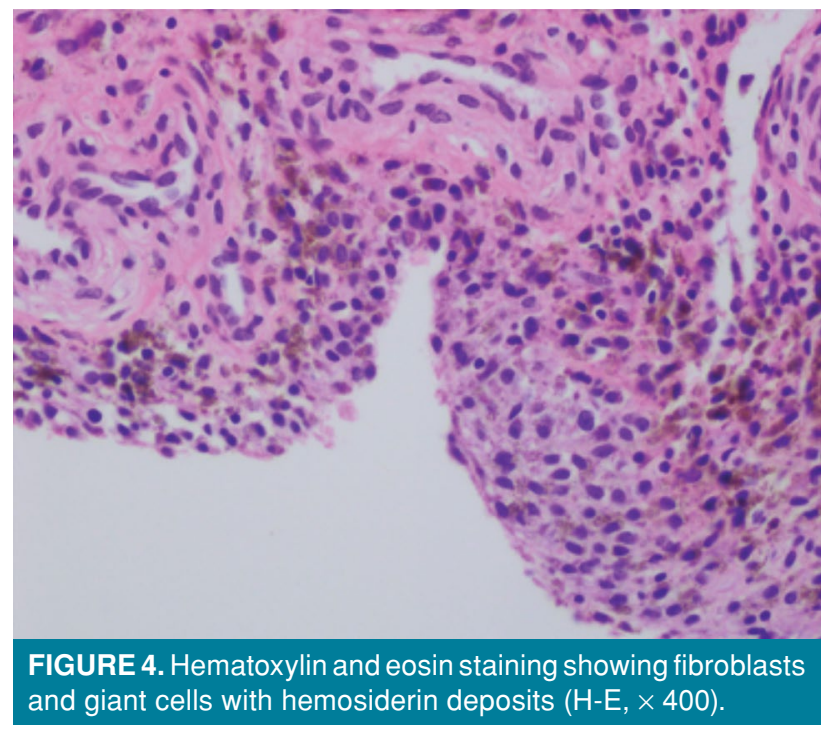

hemosiderin deposits, based on which the patient was diagnosed with localized PVNS (Figure 4). After surgery, the patient wore a Velpeau sling, and an intravenous patient-controlled analgesia (PCA) pump was connected. The pain improved immediately and there was no pain, even after removal of the intravenous PCA pump. Starting from postoperative Day 1, recovery of full ROM was achieved and all existing symptoms resolved. The plain radiograph taken on postoperative Day 1 revealed normal results with an improvement in the existing subluxation. The patient is still under follow-up and no recurrence of any specific symptoms has occurred during the 12-month follow-up.

\section{DISCUSSION}

Pigmented villonodular synovitis is also referred to as a giant cell tumor of the tendon sheath or synovial xanthoma. It is often associated with the formation of a nodular structure in the synovial bursa or synovial membrane, with hemosiderin, cholesterol, and multinuclear giant cells infiltrating the nodule, giving it a yellow to brownish color and leading to villus proliferation. Accordingly, this disease is considered a soft tissue tumor. ${ }^{[2,5]}$

Pigmented villonodular synovitis in the shoulder is a very rare disease. According to several authors, less than $3 \%$ of all PVNS cases are reported to occur in the shoulder joints. ${ }^{[6]}$ The controversy regarding the etiology of PVNS is ongoing and repetitive trauma, benign tumors of synovial fibrous histiocytoma origin, inflammation of the synovial membrane caused by an unknown stimulus, and abnormalities of fat metabolism have been proposed to play a role in its etiology. ${ }^{[7]}$ However, the exact etiology still remains unclear and, even in the present case, the patient had no history of specific trauma. The clinical symptoms of PVNS in the shoulder are non-specific and diverse. ${ }^{[8]}$ In the present case, pseudoparalysis due to inflammation and impingement caused by a relatively large intraarticular mass, approximately $3 \mathrm{~cm}$ in size, may have occurred. In a previous case reported by our group, which involved knee-locking symptoms due to PVNS originating in the meniscocapsular junction of the knee, manifestation of symptoms due to mechanical impingement was described. It is thought that these two cases share the common feature of the symptoms being caused by a similar mechanism..$^{[9]}$

Pigmented villonodular synovitis has localized and diffuse forms: the diffuse form is often an intraarticular lesion in the shoulder joint, and there have been two exceptional reports of complete extraarticular lesions. ${ }^{[10,1]}$ Localized PVNS involving the shoulder joint and biceps tendon was reported by Sipahioglu et al. ${ }^{[12]}$ In certain cases, the diffuse form is accompanied by a rotator cuff tear (RCT), which is not found in the localized form. ${ }^{[13]}$ Indeed, the MRI findings in the present case did not reveal any RCT.

For the treatment of PVNS, intralesional resection is recommended for inactive PVNS, marginal resection is recommended for active PVNS, and extensive resection is recommended for aggressive PVNS. However, this requires resection of all lesions, synovial membranes, and submucosa limbal tissue, which is realistically impossible and may leave sequelae; thus, adjuvant therapy, such as chemotherapy or radiotherapy, is used after partial resection of the synovial membrane. ${ }^{[5,13]}$ In case of the localized form, partial synovial resection has been reported to show good outcomes, along with mostly good prognosis and no recurrence in most cases. ${ }^{[8]}$ In contrast to open treatment, arthroscopic treatment of PVNS of the shoulder joint allows removal of tumors in every corner of the joint using front and rear insertion points and expansion and traction of the joint with a fluid control system. Therefore, there is an increasing trend to prefer arthroscopic treatment as anteroinferior, posterosuperior, and posteroinferior aspects of the joint capsule, which cannot be reached in an open removal procedure and can be reached arthroscopically. In the present case, arthroscopy was used to identify the lesions and site of origin of localized PVNS, while all residual areas suspected of being tumorous and peripheral and surrounding synovitis were removed using a shaver and electrocoagulation device. It is believed that 
arthroscopic treatment is a very effective treatment method for intraarticular localized PVNS. Mahieu et al. ${ }^{[13]}$ reported no recurrence after performing arthroscopic synovial resection in two cases. Mulier et al. ${ }^{[14]}$ also reported that recurrences in the shoulder were less common than in the knee, as the synovial resection could be performed more easily in the shoulder joint. In the present case, the patient has remained asymptomatic for three months after surgery, although further long-term follow-up is necessary.

In conclusion, localized PVNS of the shoulder joint should be kept in mind in patients with a suddenonset shoulder joint subluxation and pseudoparalysis.

\section{Acknowledgements}

We would like to acknowledge Kyeong-Rae Kim, a Nanoori Scientific Team member, for his valuable assistance in acquiring the full text articles for the literature review and managing digital works.

\section{Declaration of conflicting interests}

The authors declared no conflicts of interest with respect to the authorship and/or publication of this article.

\section{Funding}

The authors received no financial support for the research and/or authorship of this article.

\section{REFERENCES}

1. Myers BW, Masi AT. Pigmented villonodular synovitis and tenosynovitis: a clinical epidemiologic study of 166 cases and literature review. Medicine (Baltimore) 1980;59:223-38.

2. Müller LP, Bitzer M, Degreif J, Rommens PM. Pigmented villonodular synovitis of the shoulder: review and case report. Knee Surg Sports Traumatol Arthrosc 1999;7:249-56.
3. Sher M, Lorigan JG, Ayala AG, Libshitz HI. Case report 578: Pigmented villonodular synovitis of the shoulder. Skeletal Radiol 1990;19:131-3.

4. Chiang ER, Ma HL, Wang ST, Hung SC, Chen TH. Arthroscopic treatment for pigmented villonodular synovitis of the shoulder associated with massive rotator cuff tear. Arthroscopy 2009;25:716-21.

5. Cheng JC, Wolf EM, Chapman JE, Johnston JO. Pigmented villonodular synovitis of the shoulder after anterior capsulolabral reconstruction. Arthroscopy 1997;13:257-61.

6. Dorwart RH, Genant HK, Johnston WH, Morris JM. Pigmented villonodular synovitis of synovial joints: clinical, pathologic, and radiologic features. AJR Am J Roentgenol 1984;143:877-85.

7. Nielsen GP, Rosenberg AE, O'Connell JX, Kattapuram SV, Schiller AL. Tumors and diseases of the joint. Semin Diagn Pathol 2011;28:37-52.

8. Flandry F, Norwood LA. Pigmented villonodular synovitis of the shoulder. Orthopedics 1989;12:715-8.

9. Kim H, Shin DC, Lee KS, Jang IT, Lee K. Localized pigmented villonodular synovitis with hemorrhage arising from lateral meniscocapsular junction: A case report. Eklem Hastalik Cerrahisi 2019;30:177-81.

10. Sawmiller CJ, Turowski GA, Sterling AP, Dudrick SJ. Extraarticular pigmented villonodular synovitis of the shoulder: a case report. Clin Orthop Relat Res 1997;(335):262-7.

11. Konrath GA, Nahigian K, Kolowich P. Pigmented villonodular synovitis of the subacromial bursa. J Shoulder Elbow Surg 1997;6:400-4.

12. Sipahioğlu S, Zehir S, Aşkar H, Ozkanli U. Diffuse pigmented villonodular synovitis in the shoulder joint and the biceps tendon: a case report. Eklem Hastalik Cerrahisi 2011;22:172-6.

13. Mahieu X, Chaouat G, Blin JL, Frank A, Hardy P. Arthroscopic treatment of pigmented villonodular synovitis of the shoulder. Arthroscopy 2001;17:81-7.

14. Mulier T, Victor J, Van Den Bergh J, Fabry G. Diffuse pigmented villonodular synovitis of the shoulder. A case report \& review of literature. Acta Orthop Belg 1992;58:93-6. 